CORRECTIONS

\title{
Author's reply to Steer
}

During the editing process, a word was mistakenly omitted from the second paragraph of this Letter (BMJ 2013;347:f4868, doi:10.1136/bmj.f4868). The word "not" was erroneously removed from the second sentence, which should have read: "It is not ideal to estimate iron stores from haemoglobin concentrations or erythrocyte indices."

Cite this as: BMJ 2013;347:f5139

๑ BMJ Publishing Group Ltd 2013 